\section{Functional Significance of the C324R Mutation Examined Using a Structural Biological Approach}

\section{To the Editor:}

We read with interest in The Journal of Rheumatology the letter from Maeshima, et al, "An Adult Fatal Case with a STAT1 Gain-of-function Mutation Associated with Multiple Autoimmune Diseases"1. The authors presented the case of a 25-year-old Japanese woman, who was diagnosed at 8 years of age with autoimmune hypothyroidism, and accidentally at 21 years with Takayasu arteritis (TA). By using DNA sequencing, they identified a signal transducer and activator of transcription 1 (STAT1) gain-of-function (GOF) c.970T>C (p.C324R) mutation, in the DNA binding domain of STAT1 protein. STAT1 is one of the 7-member mammalian STAT family and latent DNA-binding protein mediating transmission from various signals to specific gene expression ${ }^{2}$. Although hypothyroidism, inflammatory bowel disease, and thrombocytopenia are common autoimmune features of STATI GOF mutations ${ }^{3}$, TA represents a previously unreported phenotype of STAT1 GOF mutations ${ }^{1}$.

Prompted by this unique finding, and considering that DNA sequencing did not reveal any evidence of common variable immunodeficiency or other candidate primary immunodeficiency diseases-associated genetic alterations in any of 44 candidate genes tested ${ }^{1}$, we attempted to elucidate further the functional significance of the C324R mutation by using a structural biological approach. The general aspects of the interface between the STAT1 DNA-binding domain and DNA suggest that relatively few direct contacts between STAT1 side chains and the DNA bases are likely to occur. This is consistent with the pattern of sequences in natural STAT-binding sites, which do not show a sharply defined consensus sequence. Rather, specificity in DNA targeting is likely to arise from interactions between the STAT dimer on DNA and other proteins, particularly other STAT dimers bound to adjacent DNA sites ${ }^{4,5}$. The 3-D structure of the tyrosine phosphorylated STAT1 dimer bound to DNA (Protein Data Bank code 1BF5) was used to analyze the consequences to structure and function of the mutation $\mathrm{C} 324 \mathrm{R}$ using molecular modeling. The electrostatic surface potential of the models was calculated by the Adaptive Poisson-Boltzmann Solver using the PyMOL plug-in with the default variable settings. The molecular graphics program PyMOL $^{6}$ was used to analyze the conformational changes caused by the mutation and to display the results.
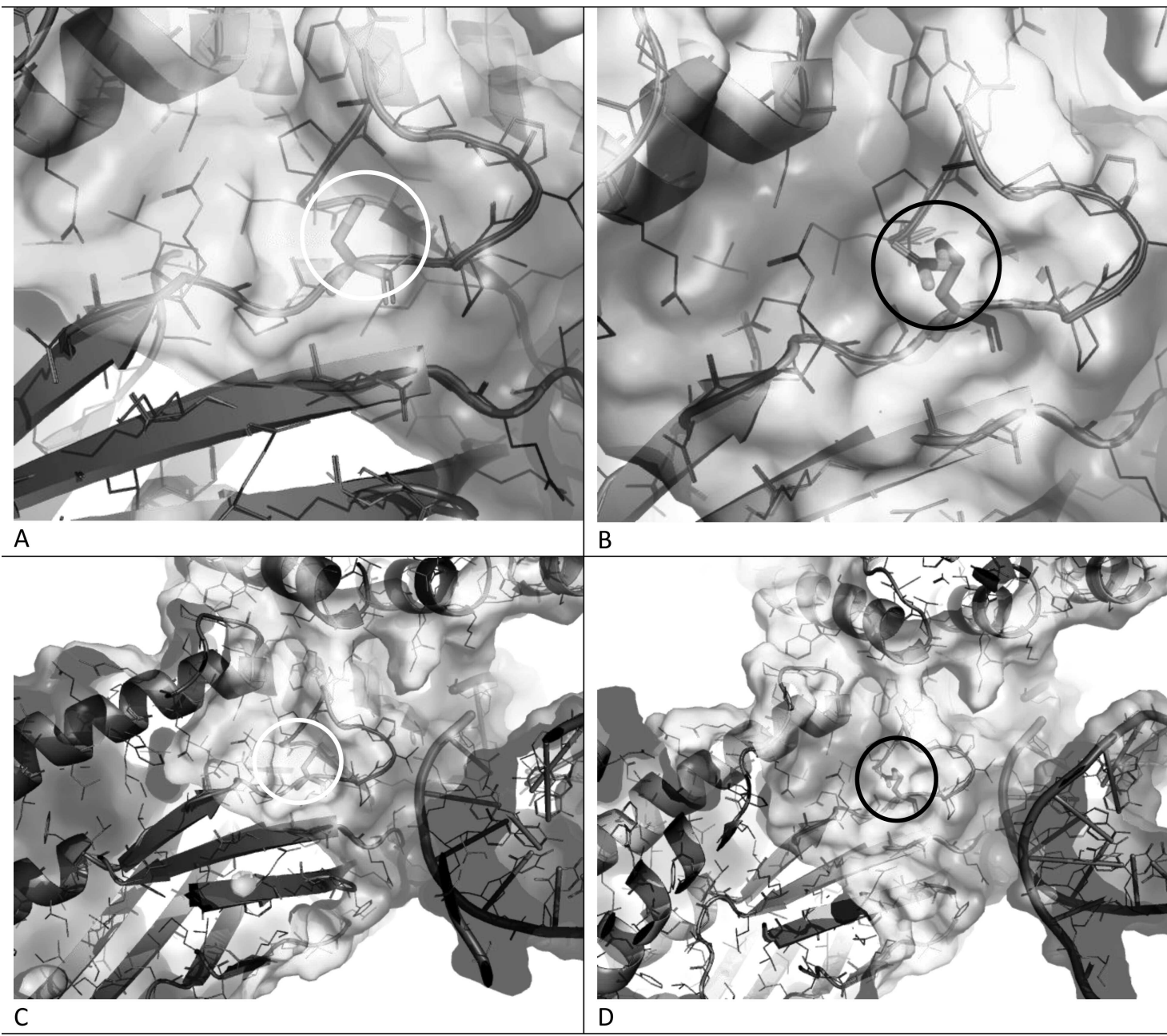

Figure 1. Electrostatic surface and ribbon representation of the native and mutant C324R 3-D models of the DNA-binding domain of STAT1 and the DNA interaction. Panels A and C are close-up and distant views from the native experimental structure (PDB code 1BF5) and panels B and D are respective views for the mutant C324R mutant. Position 324, in stick representation, is highlighted with shaded circles (white for the native cysteine and black for the arginine residues). Charge distribution is shown with a shade gradient from light (positive) to dark shade (negative). The nonlinear Poisson-Boltzmann equation is contoured at $\pm 5 \mathrm{kT} / \mathrm{e}$ electrostatic potential, depicting the negatively and positively charged surface areas colored in light and dark shade, respectively.

Personal non-commercial use only. The Journal of Rheumatology Copyright $@$ 2019. All rights reserved. 
We noted with interest that the position of the mutation C324R present in the $\mathrm{S} 1$ interacting segment of the DNA binding domain (residues $320-340)^{7}$ is located after a $\beta$ bulge and in the loop structure between strands $\beta 1$ and $\beta 2$ of the immunoglobulin-like domain (IMD) present in STAT1-4 but missing in STAT5 $\alpha$ and STAT6. Direct contact of this loop with the DNA's phosphoribose chain occurs through Thr327 in STAT1. The native IMD features a weakly positive charge distribution for this segment because of the presence of positively charged residues conserved among the STAT (Arg, Lys). The small noncharged cysteine residue at position 324 acts as a mold for the correct formation and positioning of the extensive interacting loop (325-332) restricting the conformational space distal to the surface (Figure 1A, 1C). The replacement of this position by a positively charged arginine residue introduces additional positive charge (Figure 1B, 1D), heavily enhancing the attraction and disrupting the weak nonspecific interaction to the DNA molecule. This positively charged enhancement is also altering the charge distribution toward the close neighboring coiled domain on the other side (distal to the DNA binding interface), participating in interactions with other helical proteins with specificity arising from the intercalation of complementary charges ${ }^{7}$

To our knowledge, this is the first study to evaluate the structural significance of the STAT1 GOF mutation under discussion. We conclude that, owing to the size of the arginine side chain, the loop 325-332 structure is forced to change conformation, affecting the position of the interaction with the DNA residues. As a result, a heavily modified interaction with the transcription factor is expected. This information would help to further interpret the findings of Maeshima, et al from the structural biology point of view.

GEORGE N. GOULIELMOS, PhD, Professor of Human Molecular Genetics, Section of Molecular Pathology and Human Genetics, Department of Internal Medicine, School of Medicine, University of Crete, Heraklion; MARIA I. ZERVOU, MSc, PhD, Laboratory Teaching Staff, Section of Molecular Pathology and Human Genetics, Department of Internal Medicine, School of Medicine, University of Crete, Heraklion;
ELIAS ELIOPOULOS, PhD, Professor of Biochemistry, Department of Biotechnology, Agricultural University of Athens, Athens, Greece. Address correspondence to G. Goulielmos, Section of Molecular Pathology and Human Genetics, Department of Internal Medicine, School of Medicine, University of Crete, Heraklion, Greece.

E-mail: goulielmos@med.uoc.gr

\section{REFERENCES}

1. Maeshima K, Ishi K, Shibata H. An adult fatal case with a STAT1 gain-of-function mutation associated with multiple autoimmune diseases. J Rheumatol 2019;46:325-6.

2. Reich NC. STAT dynamics. Cytokine Growth Factor Rev 2007;18:511-8.

3. Toubiana J, Okada S, Hiller J, Oleastro M, Lagos Gomez M, Aldave Becerra JC, et al; International STAT1 Gain-of-Function Study Group. Heterozygous STAT1 gain-of-function mutations underlie an unexpectedly broad clinical phenotype. Blood 2016; 127:3154-64.

4. Vinkemeier U, Cohen SL, Moarefi I, Chait BT, Kuriyan J, Darnell JE Jr. DNA binding of in vitro activated Stat1 alpha, Stat1 beta and truncated Stat 1: interaction between NH2-terminal domains stabilizes binding of two dimers to tandem DNA sites. EMBO J 1996;15:5616-26.

5. Xu X, Sun YL, Hoey T. Cooperative DNA binding and sequence-selective recognition conferred by the STAT aminoterminal domain. Science 1996;273:794-7.

6. Schrödinger LLC. The PyMOL Molecular Graphics System 2016; version 2.2. [Internet. Accessed March 5, 2019.] Available from: pymol.org/2/support.html

7. Chen X, Vinkemeier U, Zhao Y, Jeruzalmi D, Darnell JE Jr, Kuriyan J. Crystal structure of a tyrosine phosphorylated STAT-1 dimer bound to DNA. Cell 1998;93:827-39.

J Rheumatol 2019;46:6; doi:10.3899/jrheum.181346 